\title{
Enhanced antitumor efficacy by the combination of emodin and gemcitabine against human pancreatic cancer cells via downregulation of the expression of XIAP in vitro and in vivo
}

\author{
ZHAO-HONG WANG ${ }^{*}$, HUI CHEN* ${ }^{*}$ HONG-CHUN GUO, HONG-FEI TONG, JIN-XIANG LIU, \\ WEI-TIAN WEI, WEI TAN, ZHONG-LIN NI, HAI-BIN LIU and SHENG-ZHANG LIN \\ Department of Hepatobiliary-pancreatic Surgery, The Second Affiliated Hospital of Wenzhou \\ Medical College, No. 109, West Xue-yuan Road, Wenzhou 325027, P.R. China
}

Received May 13, 2011; Accepted June 19, 2011

DOI: 10.3892/ijo.2011.1115

\begin{abstract}
XIAP and NF-kB play an important role in chemotherapy resistance in pancreatic cancer. The purpose of this study was to explore the role of XIAP and NF- $\mathrm{\kappa B}$ in potentiating the antitumor effect of gemcitabine by emodin in pancreatic cancer. SW1990 cells were treated by sodium chloride, gemcitabine, emodin or their combination (gemcitabine plus emodin). Cellular proliferation and apoptosis were detected by Cell Counting kit-8 (CCK-8) assay and flow cytometry in vitro. The combination therapy more significantly inhibited SW1990 cell growth and induced a higher percentage of apoptosis than monotherapy. Gemcitabine upregulated the expression of XIAP and NF- $\mathrm{KB}$, while emodin or emodin plus gemcitabine downregulated them compared to the control group in vitro. SW1990 cells were used to establish orthotopic pancreatic tumor models in nude mice. Tumor-bearing mice were treated with sodium chloride, emodin, gemcitabine or their combination. After being treated for 4 weeks, the nude mice were imaged with high-resolution positron emission tomography (microPET) and fluorine-18-labeled fluorodeoxyglucose $\left({ }^{18} \mathrm{~F}\right.$-FDG) to detect the tumor/non-tumor ratio (T/NT ratio) and standard uptake value (SUV). The mice were sacrificed to determine tumor weight. The combination of emodin and gemcitabine showed more significant reduction in the T/NT ratio, SUV and tumor weight compared to monotherapy. The mRNA levels and the protein expression of XIAP and NF- $\kappa \mathrm{B}$ were upregulated in the gemcitabine group, while they were downregulated in the emodin group and the combination group in vivo. Ki-67 proliferation index and TUNEL assay results also showed that
\end{abstract}

Correspondence to: Dr Sheng-Zhang Lin, The Second Affiliated Hospital of Wenzhou Medical College, No. 109, West Xue-yuan Road, Wenzhou 325027, P.R. China

E-mail: wzf21sz@163.com

*Contributed equally

Key words: emodin, pancreatic cancer, gemcitabine, XIAP, NF-кB emodin enhanced tumor apoptosis induced by gemcitabine in vivo. This study suggests that emodin enhances the antitumor effect of gemcitabine in SW1990 pancreatic cancer in vitro and in vivo, which may be via the downregulation of NF- $\kappa \mathrm{B}$ expression, thus inhibiting the expression of XIAP.

\section{Introduction}

Because of the deep location, difficulties in early diagnosis, rapid progression and poor curative effect, the pancreatic cancer's 5-year survival rate is very low (1), only $<5 \%$ patients survive 5 years (2). In recent years, chemotherapy based on gemcitabine is the major treatment option, but the effective rate is low and the median survival duration is not over 6 months, the 5 -year survival rate improved only slightly $(1,3)$. There are now clinical trials on the improvement of pancreatic cancer curative effect using cisplatin-targeted drug combined with gemcitabine (4). The combination could improve the effect to some extent (4), but could not prolong the survival duration significantly (5). The newest study demonstrated that curcumin, thymoquinone $(6,7)$ combined with gemcitabine inhibited pancreatic cancer cell proliferation significantly.

Emodin (1, 3, 8-trihydroxy-6-methylanthraquinone), one of the major active principles of Rheum, has antibacterial (8), antiinflammatory (9) and immunologic suppression (10) etc effects. Recent studies demonstrated that emodin could inhibit the growth of lung cancer cells (11), ovarian cancer (12), leukemia cells (13) and enhance the inhibition effect of taxol on cancer cell proliferation. These actions were acquired through the regulation of the genes associated with tumor cell proliferation (12) and apoptosis (2,14-16). XIAP, an important member of anti-apoptosis gene family, widely exists in human tumor cells and is involved in tumor cell proliferation and anti-apoptosis. The expression of XIAP can interrupt cell apoptosis and induce infinite cell proliferation (17). Downregulation of the expression of XIAP in the Lewis lung carcinoma cells, hepatoma HepG2 and Molt-4 leukemia cells gave rise to apoptosis of cancer cells (18). An obvious downregulation in the expression of NF- $\kappa \mathrm{B}$ may be sufficient for the treatment of advanced pancreatic cancer as reported (18). Our research investigated the effect of gemcitabine plus with emodin on pancreatic cancer in vitro and 
in vivo, and the possible mechanism is that emodin enhances the antitumor effect of gemcitabine by downregulating the expression of XIAP and NF-kB.

\section{Materials and methods}

Drugs and reagents. Emodin and the terminal deoxynucleotidyl transferase-mediated deoxyuridine triphosphate nick-end labeling (TUNEL) assay was purchased from Sigma (St. Louis, MO, USA) and emodin was dissolved in dimethylsulfoxide (DMSO). The final concentration of DMSO was $<0.1 \%$. Gemcitabine was purchased from Ely Lilly Company (France) and dissolved in sterile $0.9 \%$ sodium chloride to make $0.2 \mathrm{mmol} / \mathrm{l}$ stock solution. RPMI-1640, parenzyme and fetal bovine serum (FBS) were obtained from Gibco-BRL (Grand Island, NY). The TRIzol reagent was obtained from Invitrogen (Carlsbad, CA, USA), whereas the RNA fast 200 purification kit was obtained from Fastagen Biotech (Shanghai, China). Antibodies were obtained from the following commercial sources: anti-NF- $\kappa B$ and anti- $\beta$-actin were purchased from Epitomics (Burlingame, CA, USA); anti-XIAP was obtained from Abcam (Cambridge, MA, USA); anti-Ki-67 antibody and DAB kit from Zhongshan Bio-Tech Co., Ltd. (Beijing, China). Fluorine-18-labeled fluorodeoxyglucose $\left({ }^{18} \mathrm{~F}-\mathrm{FDG}\right)$ was provided by Zhejiang University School (Hangzhou, Zhejiang, China).

Cell culture. The human pancreatic cancer cell line SW1990 was obtained from American Type Culture Collection (ATCC, USA) and grown in RPMI-1640 supplemented with $10 \%$ fetal bovine serum, 100 units $/ \mathrm{ml}$ penicillin and $100 \mathrm{mg} / \mathrm{ml}$ streptomycin in a humidified incubator containing $5 \% \mathrm{CO}_{2}$ in air at $37^{\circ} \mathrm{C}$. Refresh the nutrient fluid once per 2 3 days. Subculture: the cells were digested with tryptase when they adhered $70 \sim 80 \%$ to the culture flask bottom.

Cell survival rate detected by CCK-8. SW1990 cells collected in exponential phase were seeded at an initial density of $4 \times 10^{3}$ cells for each well in 96-well plate. They were divided into four groups: control group (0.1\% massfraction DMSO), gemcitabine group (gemcitabine $20 \mu \mathrm{mol} / \mathrm{l}$ ), emodin group (emodin $40 \mu \mathrm{mol} / \mathrm{l}$ ) and combination group (gemcitabine + emodin, gemcitabine $20 \mu \mathrm{mol} / 1$ plus emodin $40 \mu \mathrm{mol} / \mathrm{l})$, five wells for each group. Cells were treated for $24 \mathrm{~h}$. The wells were not added drugs but $0.1 \%$ massfraction DMSO as control. The blank retainer group was settled at the same time. One hour before the exposure ended, $0.01 \mathrm{ml}$ CCK-8 (Beyotime Institute of Biotechnology Haimen, China) was added into each well. A450 value was detected by ELISA (ELX800, Bio-Tek, USA). The assay was repeated three times.

Flow cytometric assessment of apoptosis. The measurement of phosphatidylserine redistribution in a plasma membrane was conducted according to the protocol outlined by the manufacturer of the Annexin V-FITC/PI apoptosis detection kit (Nanjing KeyGen Biotech. Co., Ltd., China). After 24 h treatment with different compounds, cells were harvested with $0.25 \%$ trypsin (Gibco Invitrogen Corporation, USA) and washed twice with PBS buffer. Then, cells were centrifuged at $100 \mathrm{xg}$ for $5 \mathrm{~min}$ and cell pellets were suspended in $500 \mu \mathrm{l}$ of Annexin V binding buffer $5 \mu \mathrm{l}$ of Annexin V-FITC and $5 \mu \mathrm{l}$ of PI were added and incubated with the cells for $15 \mathrm{~min}$ in dark. Binding buffer (500 $\mu \mathrm{l})$ was added to each sample, $1 \times 10^{4}$ cells were detected in every sample and the stained cells were analyzed using CellQuest software (Becton-Dickinson, NJ). The average of the results from three samples of cells for each experimental condition is presented.

Western blot analysis of XIAP and NF- $\kappa B$ expression in pancreatic cancer cells. For protein analysis, total cellular lysates were separated on SDS polyacrylamide gel and transferred to polyvinylidene fluoride. The filters were blocked in TBS with 5\% skim milk and incubated overnight with primary antibodies specific for NF- $\mathrm{BB}$ (Epitomics, Burlingame, CA, USA) and XIAP (Abcam, Cambridge, MA, USA) as a primary antibody. The filters were then incubated with anti-rabbit or anti-mouse secondary antibody conjugated with horseradish peroxidase (Santa Cruz Biotech, Santa Cruz, CA, USA). Immunoreactive bands were detected by the enhanced chemiluminescence (ECL) kit for Western blotting detection with hyper-ECL film. Equal loading was confirmed by probing the blots with $\beta$-actin antibody (Santa Cruz Biotech).

Orthotopic pancreatic carcinoma xenograft nude mouse pattern establishment. Female BALB/c nude mice, 4 6 weeks old, weight 20 25 g, were obtained (Shanghai SLAC Laboratory Animal Co. Ltd., Shanghai, China) for tumor implantation. All mice were maintained in a sterile environment under constant temperature $\left(25^{\circ} \mathrm{C}\right)$ and humidity and cared for within the laboratory animal regulations of the Ministry of Science and Technology of the People's Republic of China (http://www.most. gov.cn/kytj/kytjzcwj/200411/t20041108_32465.htm).

The food, water and bedding for these immunocompromised mice were sterilized and changed at least once weekly. A total of 40 mice were employed in the research. SW1990 pancreatic cancer cells $\left(5 \times 10^{6}\right)$ collected in $50 \mu$ l serum-free 1640 media per mouse in log phase growth were orthotopically implanted in anesthetized athymic nude mice. Briefly, a small left abdominal flank incision was made and the spleen exteriorized. SW1990 cells were injected subcapsularly in a region of the pancreas just beneath the spleen. A successful subcapsular intrapancreatic injection of cancer cells were identified by the appearance of a fluid bleb without i.p. leakage (19). All surgical procedures were done under sterilized conditions with a 10x microscope. One week after implantation, mice were medially randomized into four groups to receive intraperitoneal (i.p.) injections of vehicle ( $0.9 \%$ sodium chloride), emodin ( $40 \mathrm{mg} / \mathrm{kg}$ ) (20), gemcitabine (125 mg/kg) (21), or their combination (emodin $40 \mathrm{mg} / \mathrm{kg}$ plus gemcitabine $125 \mathrm{mg} / \mathrm{kg}$ ) three times per week for 2 weeks.

MicroPET imaging. MicroPET imaging was performed when the nude mice were treated 4 weeks later. Mice were fasted for at least $8 \mathrm{~h}$ before the imaging, $0.1 \mathrm{mCi}{ }^{18} \mathrm{~F}-\mathrm{FDG} /$ mouse was injected into the tail vein. Mice were anesthetized using $2 \%$ isoflurane and positioned in a prone position along the long axis of the microPET scanner and imaged. A 10 min data collection was performed in ${ }^{18}$ F-FDG-PET with an uptake time of $1 \mathrm{~h}$ after the tracer injection. Static acquisition was performed in three-dimensional mode using a microPET imaging system (R4, Concorde Microsystems, Knoxville, TN, USA). For quantitative evaluation, the regions of interest (ROIs) method and standard uptake value (SUV) were used to evaluate the regional uptake of the tracers. MicroPET ASIPro6.0.5.0 (Concorde Microsystems, 
Table I. Primer pairs used in reverse transcription polymerase chain reaction.

\begin{tabular}{ll}
\hline & \multicolumn{2}{c}{ Primer pairs } \\
\hline NF-кB & \\
Sense & 5'-AGC ACA GAT ACC ACC AAG ACC C-3' \\
Antisense & 5'-CCC ACG CTG CTC TTC TAT AGG AAC-3' \\
XIAP & \\
Sense & 5'-TTCCTCGGGTATATGGTGTCTGAT-3' \\
Antisense & 5'-CCGTGCGGTGCTTTAGTTGT-3' \\
GAPDH & \\
Sense & 5'-AAC GGA TTT GGT CGT ATT GGG-3' \\
Antisense & 5'-TCG CTC CTG GAA GAT GGT GAT-3' \\
\hline
\end{tabular}

XIAP, X-linked inhibitor of apoptosis protein; NF- $\mathrm{B}$, nuclear factor- $\kappa \mathrm{B}$; GAPDH, glyceraldehyde-3-phosphate dehydrogenase.

Inc., Knoxville, TN) was used in the statistical analyses for ROIs and SUVs. ROIs were drawn around the tumors and surrounding areas on the coronal slices that showed the best delineation of the tumors. All images were displayed on the same color scale, shown in Fig. 3. The highest uptake (percentage injection dose) within the tumor ROI and the mean uptake (percentage injection dose) in the surrounding ROI were recorded and calculated, respectively. $\mathrm{T} / \mathrm{N}$ ratio was calculated for the semiquantitative analysis using the following formula: $\mathrm{T} / \mathrm{N}$ ratio = pixel maximum uptake in tumor ROI/pixel mean uptake in surrounding ROI (3). $\mathrm{T} / \mathrm{N}$ ratio stands for tumor growth metabolism. The SUVs for ROIs at different groups were compared. SUV = concentration of radioactivity in the $\mathrm{ROI}(\mathrm{mCi} / \mathrm{ml}) \mathrm{x}$ body weight $(\mathrm{g}) /$ injected dose $(\mathrm{mCi})$. At the end of the experiment, mice were sacrificed by cervical dislocation after the last PET imaging one week later. Tumor samples were weighed, preserved as freshly frozen in liquid nitrogen and in $10 \%$ formalin before processing.

TUNEL assay detect tumor apoptosis. We assessed the degree of tumor apoptosis with the TUNEL method after the nude mice were sacrificed. TUNEL staining of paraffin-embedded tumor sections was done with the TUNEL kit according to the manufacturer's instructions. Laser scanning confocal microscope (Olympus BX51, Japan) under 400-fold observation camera was used, with excitation wavelength $488 \mathrm{~nm}$ and emission wavelength $568 \mathrm{~nm}$. We observed 10 field visions of the strongest fluorescence on each slice.

Immunohistochemistry detection of apoptosis-related proteins: $N F-\kappa B, X I A P$ and $K i-67$. Formalin-fixed, paraffin-embedded tumor tissues were sectioned and blocked with goat serum and immunostained after deparaffinization and rehydration. Sections were incubated with anti-human anti-NF- $\mathrm{B}$, -XIAP or -Ki-67 primary antibodies in a moist chamber overnight at $4^{\circ} \mathrm{C}$. After phosphate-buffered saline (PBS) rinses, specimens were incubated for another $30 \mathrm{~min}$ at room temperature with horseradish peroxidase (HRP)-conjugated secondary antibody. After staining with hematoxylin, sections were mounted and evaluated via microscopy (Olympus BX51, Japan). Non-specific primary antibody staining was evaluated by substitution of the primary antibody with PBS. At least 10 fields were randomly selected from each section. Images were analyzed by Image-Pro Plus 6.0 software.

Western blot analysis of XIAP and $N F-\kappa B$ expression in pancreatic cancer tissue. Pancreatic tumor xenografts were resected from the nude mice. Total protein was isolated by conventional method. Total protein concentration was measured by BCA assay kit. $80 \mu \mathrm{g}$ protein for each tissue was subjected to $12 \%$ SDS-PAGE, $80 \mathrm{~V}$ for $30 \mathrm{~min}$, then $125 \mathrm{~V}$ for $1 \mathrm{~h}$, and transferred to polyvinylene fluoride membranes. The membranes were blocked with 5\% non-fat milk TBST and incubated with the rabbit anti-human antibody overnight at $4^{\circ} \mathrm{C}$ and incubated with goat anti-rabbit IgG antibody for $1 \mathrm{~h}$. We used ECL for color development.

RT-PCR was performed to examine mRNA levels of XIAP and $N F-\kappa B$. Total RNA in tumor tissues was extracted using TRIzol reagent according to the manufacturer's instructions and purified using an RNAfast-200 purification kit. The RNA was quantified by spectrophotometric analysis and RNA integrity was confirmed by agarose gel electrophoresis. The cDNA was synthesised with the iScript cDNA synthesis kit (Bio-Rad) using $2 \mu \mathrm{g}$ RNA. The cDNA product was diluted 1:50 in deionized water, whereas primers specific for $\mathrm{NF}-\kappa \mathrm{B}, \mathrm{XIAP}$ and the GAPDH control were adjusted to $10 \mathrm{pmol} / \mu 1$ working stock with deionized water. Reverse transcription-polymerase chain reaction (RT-PCR) was conducted with the diluted cDNA and primers following the protocol outlined in the iTag SYRB Green Supermix with ROX kit (Bio-Rad) and using an ABI Prism 7700 (Applied Biosystems, Foster City, CA, USA). The amplification reaction was carried out with $1 \mu \mathrm{l}$ cDNA product for 30 cycles. The RT-PCR products were visualized on $2 \%$ agarose gels with ethidiumbromide staining under UV transillumination. The grey value was analysed using QuantityOne version 4.5 software (Bio-Rad). The primers used are listed in Table I.

Statistical analysis. Data are presented as the means \pm standard error of the mean. SPSS 13.0 was used for statistical analysis. Differences in mean values between groups were initially subjected to one-way ANOVA and then later compared among groups using unpaired Student's t-test and $\mathrm{P}<0.05$ was considered statistically significant.

\section{Results}

The effects of gemcitabine, or/and emodin on proliferation of SW1990 pancreatic cancer cells. (Fig. 1A) After the exposure of SW1990 cells to gemcitabine $(20 \mu \mathrm{mol} / \mathrm{l})$, or/and emodin (40 $\mu \mathrm{mol} / \mathrm{l})$, the cell survival rates, detected by CCK-8, were $(82.16 \pm 2.84) \%,(79.39 \pm 3.24) \%$, and $(61 \pm 3.15) \%$. The difference between combination treatment group and other monotherapy group was significant $(\mathrm{P}<0.05)$. The combination therapy resulted in more loss of cell viability than monotherapy in pancreatic cancer cells.

The effects of gemcitabine, or/and emodin on apoptosis of SW1990 pancreatic cancer cells (Fig. 1B). The result of flow cytometry indicated that SW1990 cell apoptosis rate was (24.6 \pm 3.31$) \%$ in combination group (gemcitabine $20 \mu \mathrm{mol} / 1 \mathrm{plus}$ emodin $40 \mu \mathrm{mol} / \mathrm{l}),(10.1 \pm 3.41) \%$ in emodin group $(40 \mu \mathrm{mol} / \mathrm{l})$, 


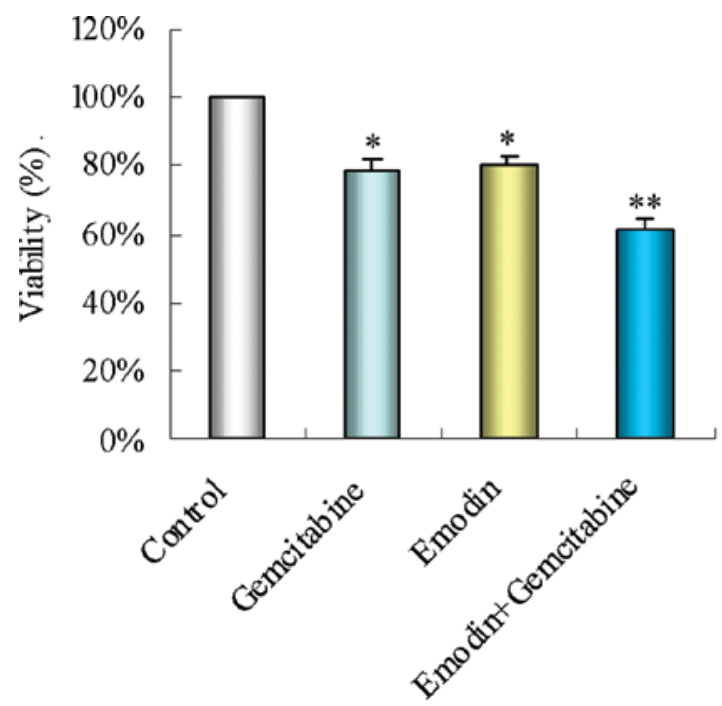

(A)

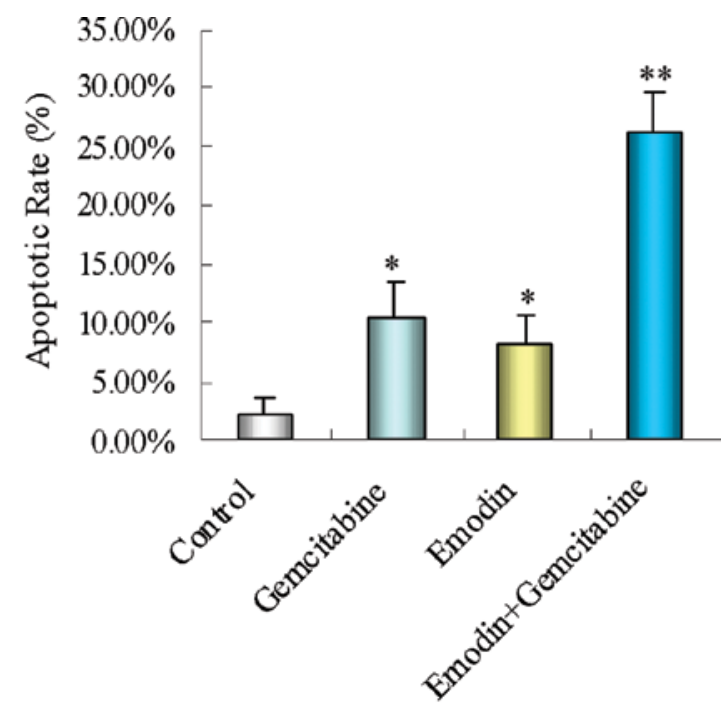

(B)

Figure 1. SW1990 cells were treated with gemcitabine $(20 \mu \mathrm{mol} / \mathrm{l})$, emodin $(40 \mu \mathrm{mol} / \mathrm{l})$ alone or their combination (gemcitabine, $20 \mu \mathrm{mol} / 1+\mathrm{emodin}, 40 \mu \mathrm{mol} / \mathrm{l})$ for $24 \mathrm{~h}$, with the untreated cells used as the control. (A) The cellular proliferation was detected by CCK-8 assay. Compared with the other groups, the cell survival rate of the combination group was significantly lower. (B) After treatment, cell apoptosis was detected by flow cytometry. Emodin combined with gemcitabine induced a higher percentage of apoptosis in pancreatic cancer cells than that of emodin or gemcitabine alone. ${ }^{*} \mathrm{P}<0.05$ compared to the control group, ${ }^{* *} \mathrm{P}<0.05$ compared to the other groups.

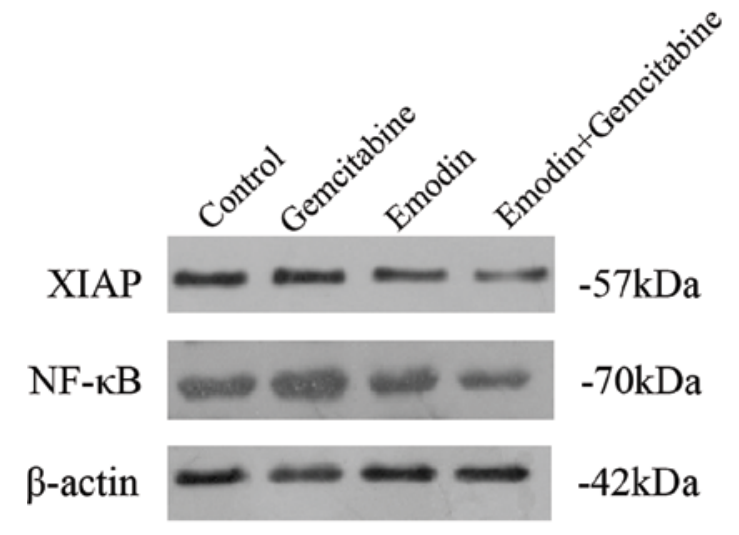

(A)

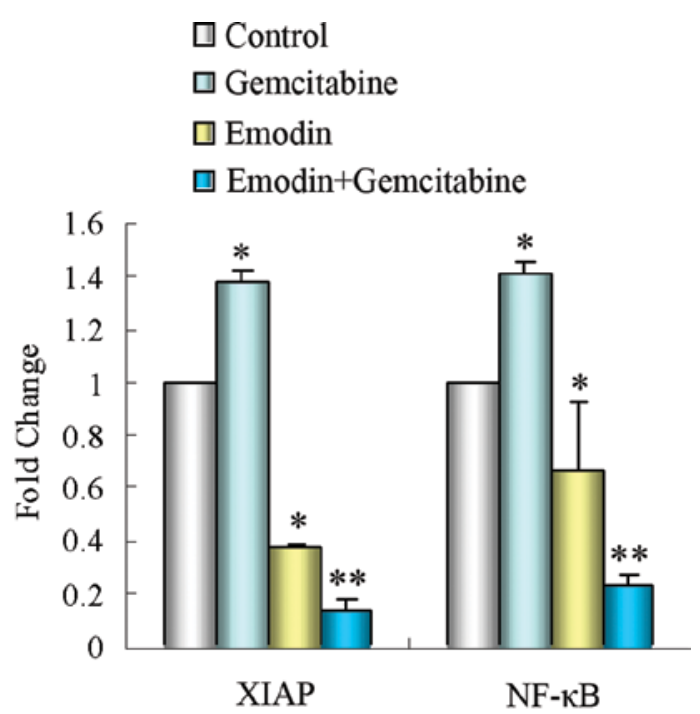

(B)

Figure 2. The protein expression of XIAP and NF- $\kappa \mathrm{B}$ were detected by Western blotting. Gemcitabine upregulated the expression level of XIAP and NF- $\kappa \mathrm{B}$ compared with control group, while emodin and their combination downregulated their expression much more significantly in vitro (A and $\mathrm{B}$ ). $\mathrm{P}<0.05$ compared to the control group, ${ }^{* *} \mathrm{P}<0.05$ compared to the other groups.

$(8.1 \pm 2.47) \%$ in gemcitabine group $(20 \mu \mathrm{mol} / \mathrm{l})$ and $(2.4 \pm 1.34) \%$ in the control group ( $0.1 \%$ massfraction DMSO). We observed emodin sensitized SW1990 cells to gemcitabine-induced apoptosis in vitro $(\mathrm{P}<0.05)$.

Western blotting was used to detect the protein expression of

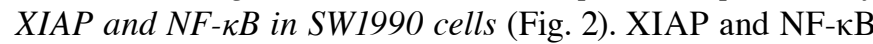
level in gemcitabine group was significantly upregulated compared with control group, while decreased in emodin group and downregulated much more significantly in the combination therapy group in vitro $(\mathrm{P}<0.05)$.

MicroPET imaging result. The survival rate was $100 \%$ (Fig. 3A). All tumors could be observed in MicroPET in coronal plane. Arrows show tumor localization (Fig. 3B). $\mathrm{T} / \mathrm{NT}$ ratio in gemcitabine group (gemcitabine $125 \mathrm{mg} / \mathrm{kg}$ ) (1.65 \pm 0.077$)$, emodin group (emodin $40 \mathrm{mg} / \mathrm{kg})(2.23 \pm 0.26)$ and gemcitabine + emodin group (gemcitabine $125 \mathrm{mg} / \mathrm{kg}$ 


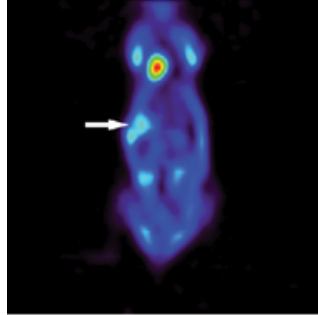

Control

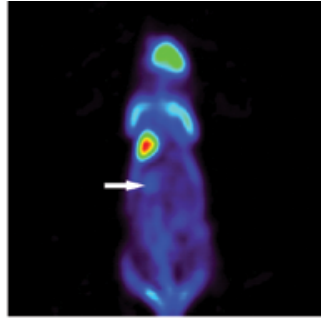

Gemcitabine

(A)

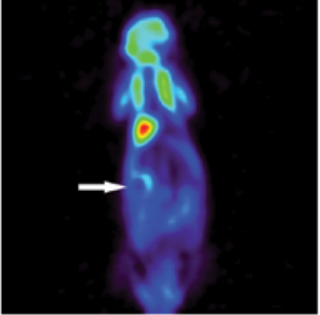

Emodin

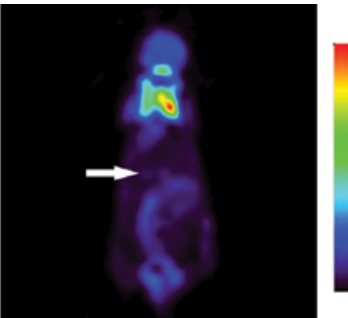

Emodin+Gemcitabine

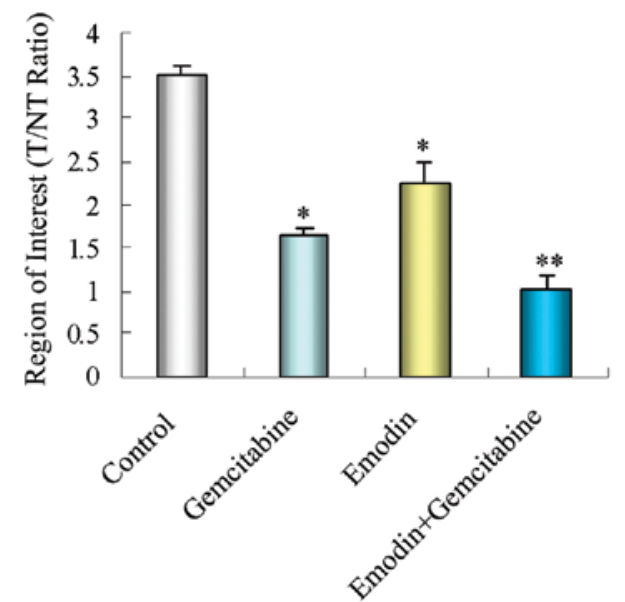

(B)

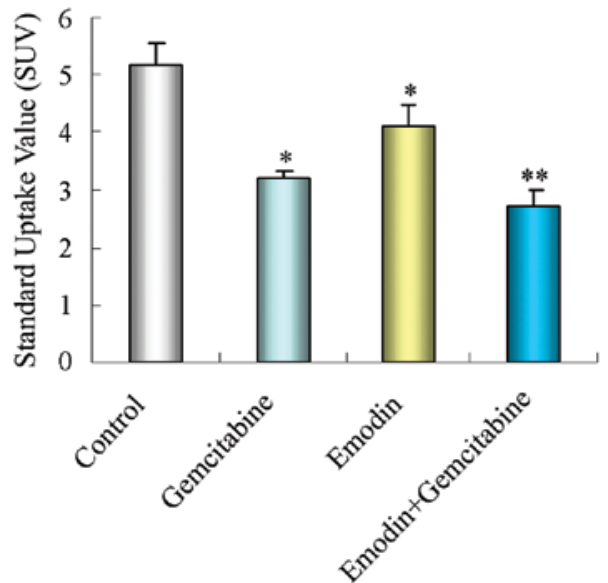

(C)

Figure 3. SW1990 cells were used to establish an orthotopic pancreatic carcinoma xenograft animal model. (A) MicroPET showing a large coronal sectional orthotopic transplantation tumor of a nude mouse. (B and C) T/NT ratio and SUVs in gemcitabine group, emodin group and combination group were less than that in control group $(0.9 \%$ sodium chloride $)(\mathrm{P}<0.05)$, while the combination group was more significant than emodin or gemcitabine group. ${ }^{*} \mathrm{P}<0.05$ compared to the control group, ${ }^{* *} \mathrm{P}<0.05$ compared to the other group.

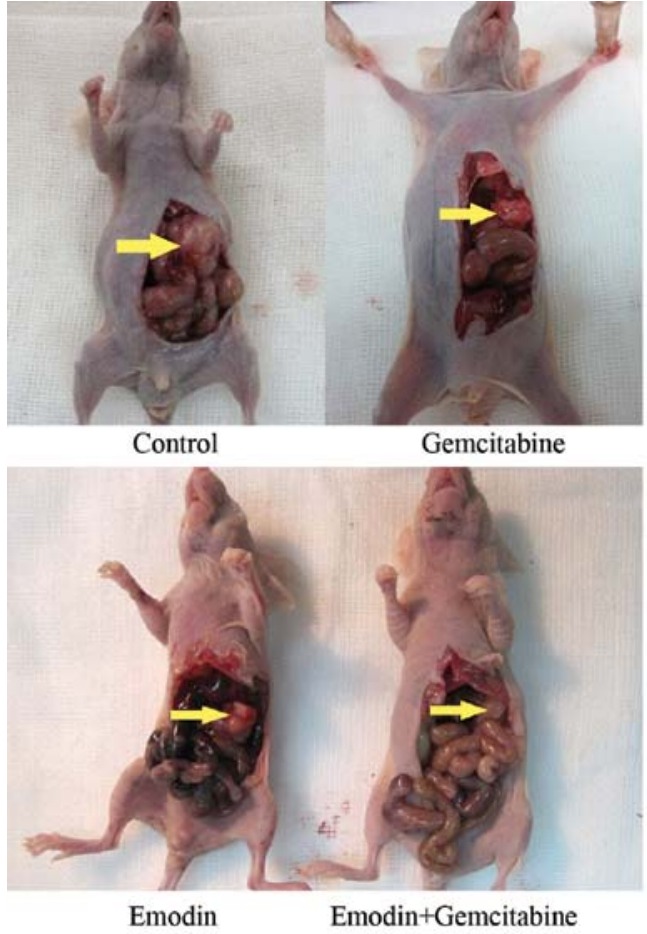

(A)

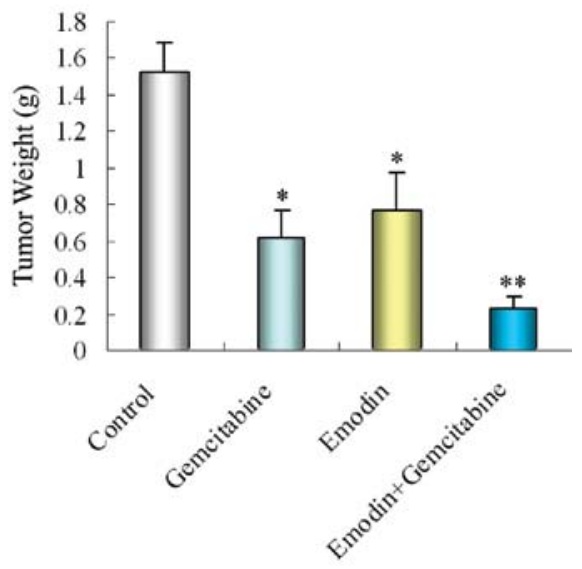

(B)

Figure 4. (A) The weight of orthotopic transplantation tumors was examined after the mice were sacrificed. (B) The weight of tumors in the different groups. ${ }^{*} \mathrm{P}<0.05$ compared to the control group, ${ }^{* *} \mathrm{P}<0.05$ compared to the other group. 


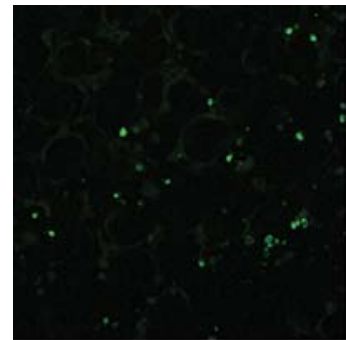

Control

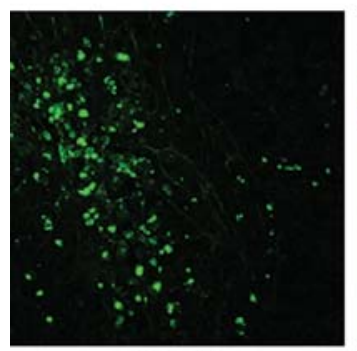

Emodin

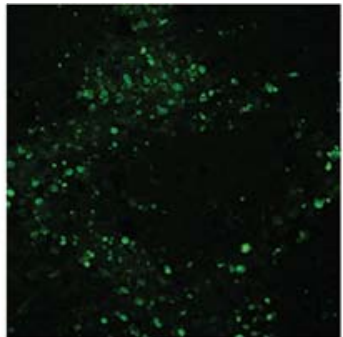

Gemcitabine

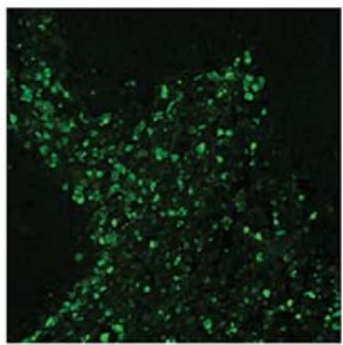

Emodin+Gemcitabine

(A)

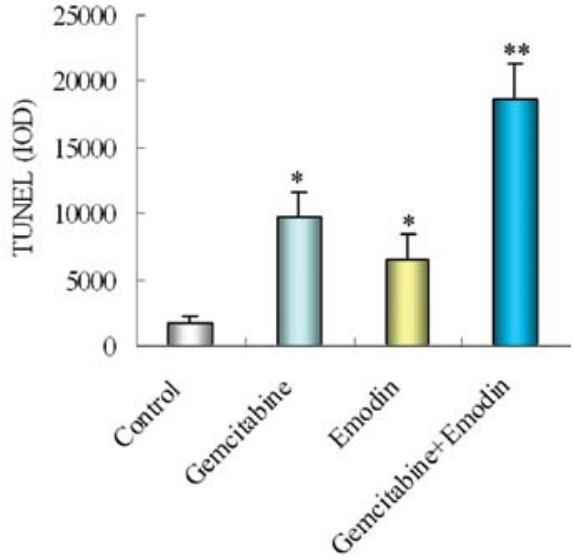

(B)

Figure 5. (A) TUNEL-positive cells have an overlay of green fluorescence from the TUNEL stain (x400). (B) TUNEL staining was further quantified and presented as average IOD level. Data are presented as the means \pm SD. ${ }^{*} \mathrm{P}<0.05$ compared to the control group; ${ }^{* * *} \mathrm{P}<0.05$ compared to the other groups.
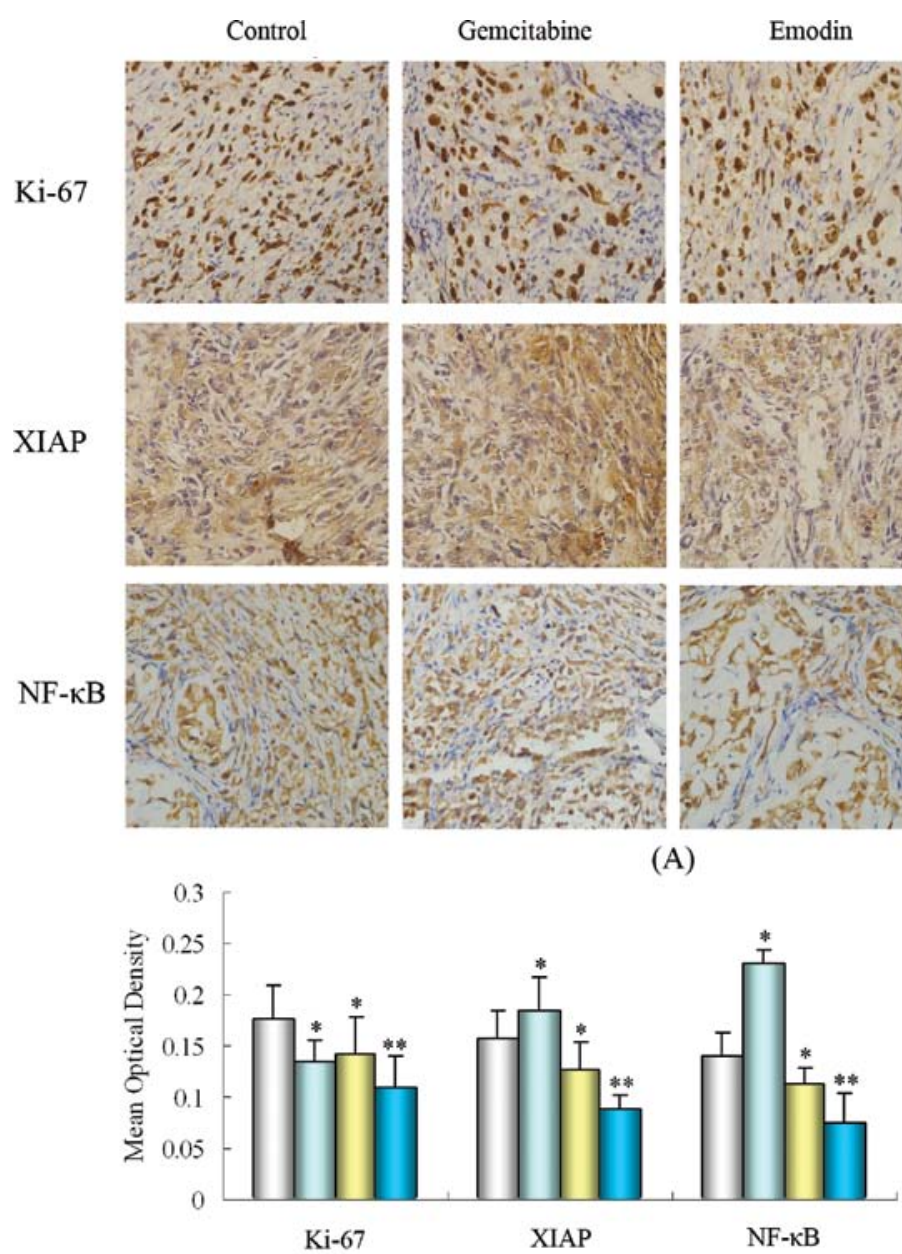

(B)

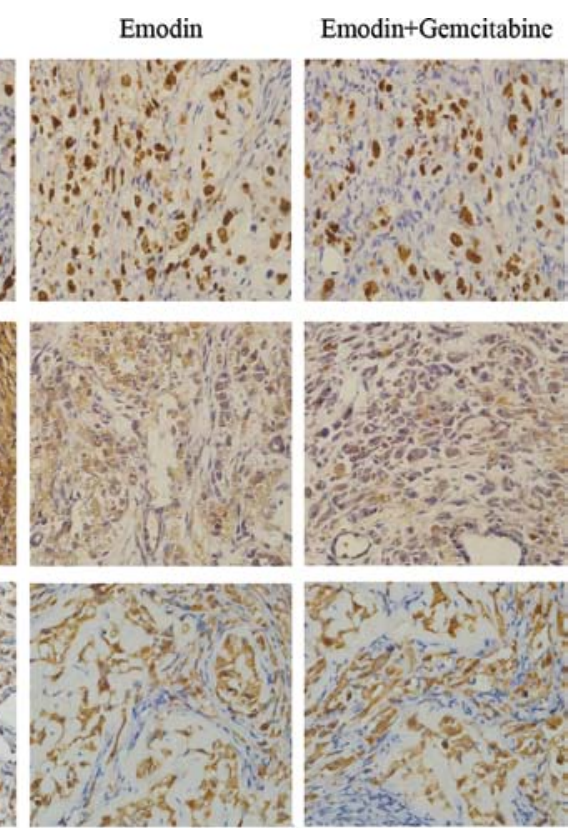

(A)
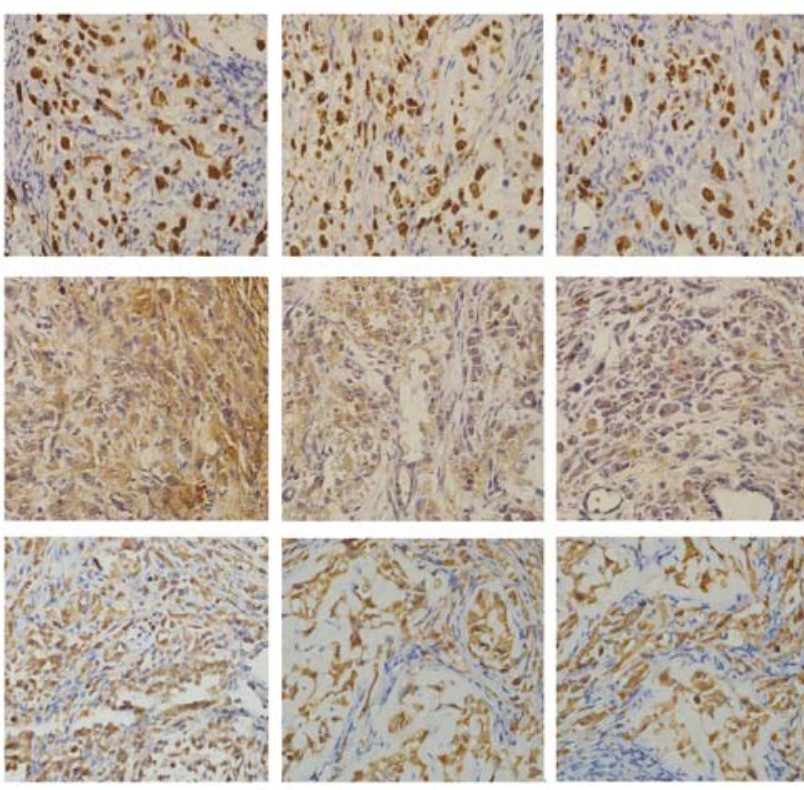

口Control

口Gemcitabine

$\square$ Emodin

口Emodin+Gemcitabine

Figure 6. The expression of Ki-67, NF- $\mathrm{kB}$ and XIAP detected by immunohistochemistry (x400). Mean optical density of Ki-67, XIAP and NF-kB in orthotopic transplantation tumor. Ki-67 indicates the inhibition of pancreatic cancer cell proliferation in emodin alone or in combination with gemcitabine-treated groups of animals. XIAP and NF- $\mathrm{BB}$ were upregulated by gemcitabine treatment, while being downregulated by emodin and combination treatment (A and B). ${ }^{*} \mathrm{P}<0.05$ compared to the control group; ${ }^{* *} \mathrm{P}<0.05$ compared to the other groups. 


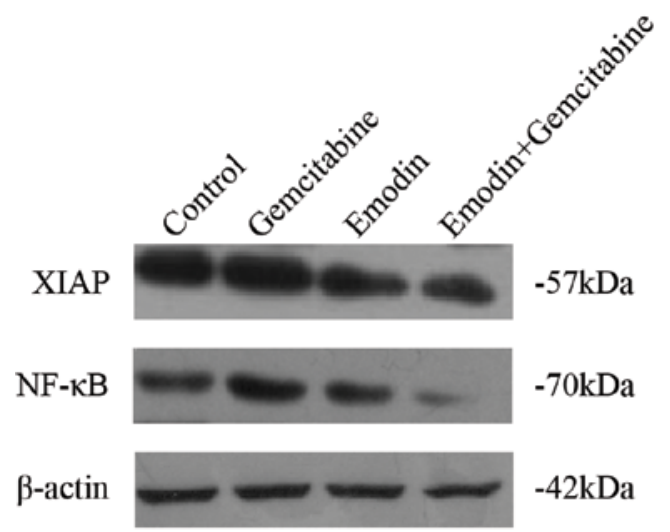

(A)

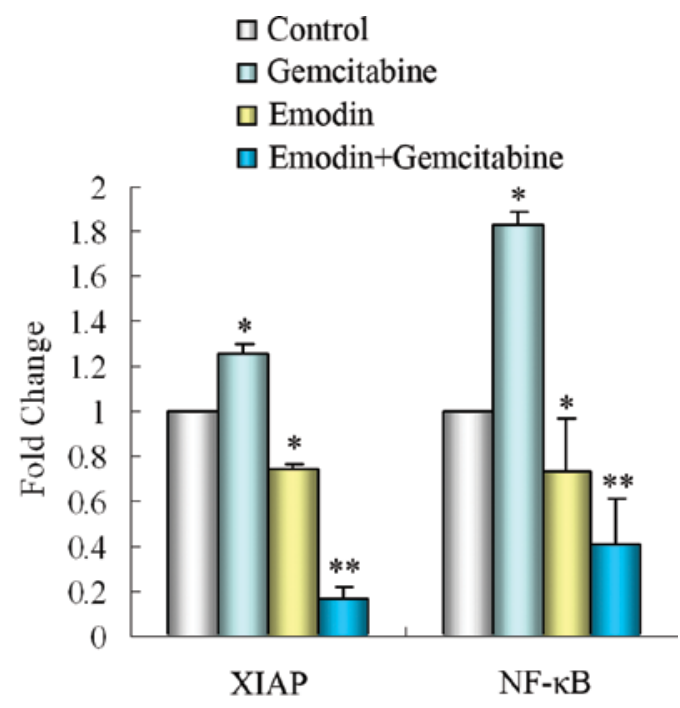

(B)

Figure 7. The protein expression of XIAP and NF- $\kappa \mathrm{B}$ in tumor tissue was detected by Western blotting in control group, gemcitabine group, emodin group and combination group. (A and B) Gemcitabine upregulated the expression of XIAP and NF- $\mathrm{B}$ level compared with control group, while emodin and their combination downregulated their expression much more significantly in vivo. ${ }^{*} \mathrm{P}<0.05$ compared to the control group, ${ }^{* *} \mathrm{P}<0.05$ compared to other groups.

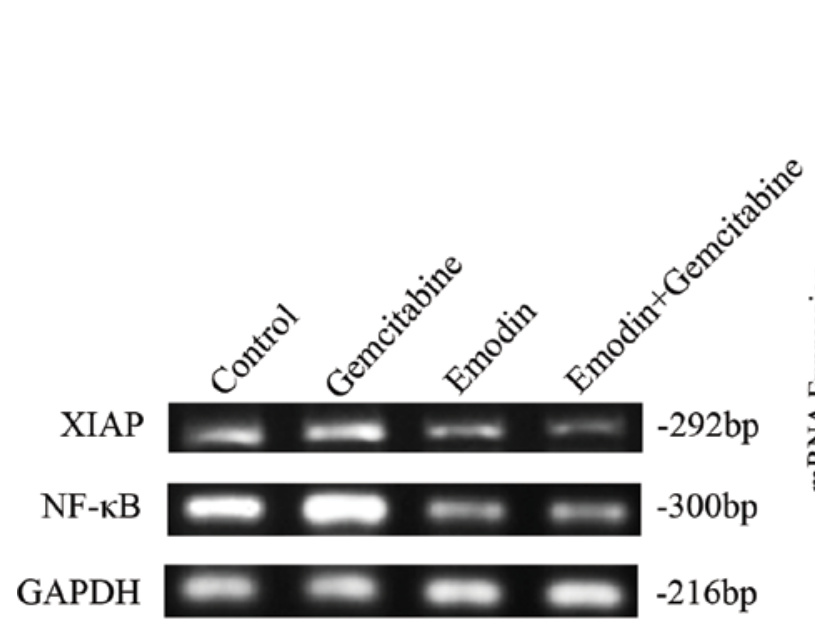

(A)

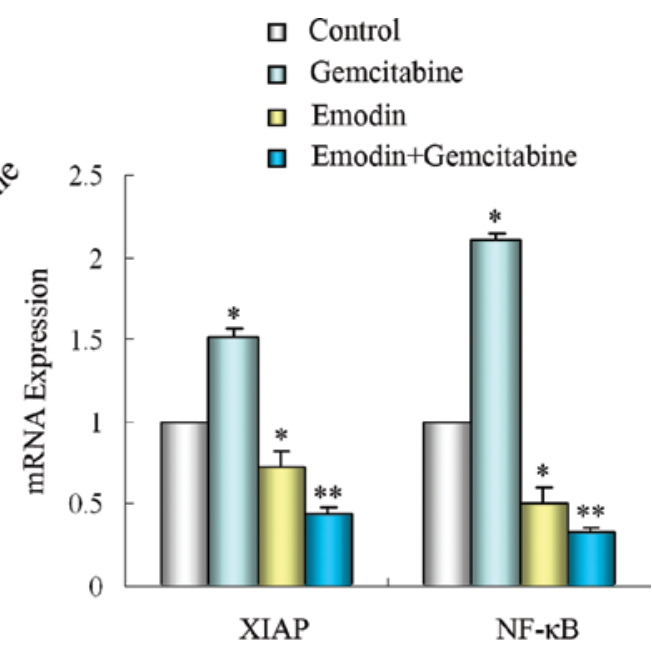

(B)

Figure 8. (A) The mRNA levels of XIAP and NF- $\mathrm{kB}$ in tumor tissue in different treatment groups were detected by RT-PCR. (B) Quantified data were presented. ${ }^{*} \mathrm{P}<0.05$ compared to the control group, ${ }^{* *} \mathrm{P}<0.05$ compared to other groups.

combined with emodin $40 \mathrm{mg} / \mathrm{kg})(1.01 \pm 0.153)$ were less than that in control group ( $0.9 \%$ sodium chloride) $(3.52 \pm 0.095)(\mathrm{P}<0.05)$ (Fig. 3C). The SUVs in different groups were: control group (5.21 \pm 0.33$)$, gemcitabine group $(3.20 \pm 0.14)$, emodin group $(4.11 \pm 0.36)$, and combination group $(2.72 \pm 0.27)$. A significant difference was found between the combination group and the other monotherapy group $(\mathrm{P}<0.05)$. The regional mean uptake of the tracers in combination therapy group was downregulated compared to monotherapy in vivo.

The inhibition effect of gemcitabine, emodin and their combination on the orthotopic transplantation tumor (Fig. 4A). After mice were sacrificed, the orthotopic transplantation tumor weight was detected (Fig. 4B). The mean weight of tumors in gemcitabine group, emodin group and combination group were respectively $(0.62 \pm 0.15) \mathrm{g},(0.77 \pm 0.20) \mathrm{g}$ and $(0.23 \pm 0.07) \mathrm{g}$, compared to the control group (1.52 \pm 0.17$) \mathrm{g}$, emodin and gemcitabine inhibited the growth of tumor in vivo, and the combination therapy was more effective $(\mathrm{P}<0.05)$.

TUNEL staining shows emodin potentiated tumor cell apoptosis by gemcitabine. Apoptosis of the tumor cells was demonstrated by TUNEL assay. TUNEL-positive cells were observed with laser scanning confocal microscope $(x 400)$ (Fig. 5). The gemcitabine $(125 \mathrm{mg} / \mathrm{kg})$ group and emodin 
( $40 \mathrm{mg} / \mathrm{kg}$ ) group promoted apoptosis of tumor cells compared to the control group. The combination therapy markedly increased the number of $\mathrm{TUNEL}^{+}$apoptotic cells compared to the other monotherapy group $(\mathrm{P}<0.05)$.

Immunohistochemistry examination of the expression of Ki-67, $X I A P$ and $N F-\kappa B$ in tumor tissue (Fig. 6). Ki-67 indicates the inhibition of pancreatic cancer cell proliferation in treatment group. The expression of Ki-67, XIAP and NF- $\mathrm{KB}$ were examined through microscope $(\mathrm{x} 400)$. Gemcitabine upregulated the expression of XIAP and NF- $\mathrm{KB}$ and downregulated the expression of $\mathrm{Ki}-67$ compared to control group in tumor tissue $(\mathrm{P}<0.05)$. Emodin single, or combined with gemcitabine significantly inhibited the expression of Ki-67, XIAP and NF- $\mathrm{KB}$ in tumor tissue compared to the control $(\mathrm{P}<0.05)$.

Western blot analysis was used to detect the protein expression of XIAP and NF- $\kappa B$ in pancreatic cancer tissue (Fig. 7). Western blotting showed that gemcitabine $(125 \mathrm{mg} / \mathrm{kg})$ upregulated the expression of XIAP and NF- $\mathrm{KB}$ in contrast to the control group in pancreatic cancer tissue $(\mathrm{P}<0.05)$; Emodin $(40 \mathrm{mg} / \mathrm{kg})$ single or combined with gemcitabine $(125 \mathrm{mg} / \mathrm{kg})$ significantly inhibited the expression of XIAP and NF- $\mathrm{KB}$ in tumor tissue compared to the control.

The mRNA levels of XIAP and NF- $\kappa B$ in pancreatic cancer tissue were detected by RT-PCR (Fig. 8). XIAP and NF- $\mathrm{KB}$ expression in pancreatic cancer tissue were upregulated in gemcitabine group, while being downregulated in emodin group and combination group in contrast to the control group in pancreatic cancer tissue $(\mathrm{P}<0.05)$.

\section{Discussion}

Natural medicine can enhance the effect of traditional chemotherapeutic drugs. Emodin was reported to enhance anti-tumor effect of paclitaxel (12). In our study, we used emodin alone or in combination with gemcitabine to treat SW1990 pancreatic cancer cell line in vitro. Degree of cellular viability and apoptosis were detected. The result showed that emodin alone or in combination with gemcitabine not only inhibited SW1990 pancreatic cancer cell proliferation, but also could promote tumor cell apoptosis, and the effect of combined treatment was more significant than the other monotherapy group. We concluded that emodin enhanced the inhibition of gemcitabine on SW1990 pancreatic cancer cells by the induction of cell apoptosis. Therefore, we established an orthotopic pancreatic tumor model in nude mice, and the growth of pancreatic tumors were more close to the physiological environment in humans. ${ }^{18}$ F-FDG-PET has a high sensitivity and specificity in detecting pancreatic carcinoma in patients with a suspiciouslooking pancreatic mass. The nude mice were treated for 4 weeks, MicroPET imaging was performed to detecte uptake of the tumor to ${ }^{18} \mathrm{~F}-\mathrm{FDG}$. The regions of interest (ROIs) method and standard uptake value (SUV) were used to evaluate the regional uptake of the tracers. We found that all treatment groups could reduce the growth metabolism of tumors and the role of combination group was much more significant $(\mathrm{P}<0.05)$, which was consistent with the results in vitro. After the mice were sacrificed, differences in the tumor weight confirmed the result. TUNEL-positive cells and $\mathrm{Ki}-67^{-}$cells were much more frequent in tumors of combination group than the other group, suggesting that emodin enhanced the effect of gemcitabine by induction of cell apoptosis.

Our research also found that gemcitabine upregulated the expression of XIAP and NF- $\mathrm{kB}$ in contrast to control group on the mRNA and protein level, while emodin single or combined with gemcitabine could significantly inhibited the expression of XIAP and NF- $\mathrm{kB}$ in pancreatic cancer cells.

$\mathrm{NF}-\kappa \mathrm{B}$ is widely expressed in mammalian cells. By regulating genes expression, $\mathrm{NF}-\mathrm{\kappa B}$ regulates cell proliferation, differentiation, apoptosis and cancerization and plays an important role in cancer occurrence and progression (23). The study of Wang et al showed that, in 24 pancreatic cancer tissues, p56, the subunit of NF- $\mathrm{BB}$, was expressed in 16 cases (67\%), while it was absent in normal pancreatic tissues (24). Our experiment also demonstrated that NF- $\mathrm{kB}$ was expressed in pancreatic cancer cells. It was reported that pancreatic cancer growth can be suppressed by inhibiting the expression of NF-kB (25). Gemcitabine has been found to upregulate the expression of NF- $\kappa \mathrm{B}$, while emodin inhibited the expression of NF-kB (26). It was demonstrated that emodin could downregulate not only the intrinsic NF- $\mathrm{KB}$ level (25), but also the expression of NF- $\kappa B$ induced by TNF (17). Our study tested the mRNA levels of NF- $\kappa B$ in pancreatic tissues by RT-PCR method. The results showed that the mRNA level of NF- $\kappa B$ in gemcitabine treatment group was upregulated, while in emodin treatment group was downregulated and in combination treatment goup was downregulated more significantly. In vitro and in vivo the protein expression was tested by Western blotting demonstrating that gemcitabine used independently could upregulate protein expression level of NF- $\mathrm{KB}$ while emodin single or combined with gemcitabine downregulated it significantly. Emodin in combination with gemcitabine was more effective than emodin alone on suppressing the expression of NF- $\mathrm{KB}$. Thus, possibly, emodin enhanced antitumor effect of gemcitabine on pancreatic cancer by inhibiting NF- $\kappa \mathrm{B}$ expression on protein and the gene.

IAPs family is one of the apoptosis-regulating genes intensively studied at present. The major biological function of IAPs is suppressing the cell apoptosis process. IAPs are highly expressed in most human malignancies. Human XIAP, a typical IAP, can interrupt cell apoptosis and urge the cells to proliferate infinitely (25). There are reports that gemcitabine can upregulate survivin, another important member of the IAP family, expression in pancreatic cancer. Emodin alone or combined with gemcitabine can downregulate the expression of survivin in pancreatic cancer significantly (27). Therefore we suspected that gemcitabine would be able to upregulate the XIAP expression in pancreatic cancer. Our experimental results indicated that gemcitabine upregulated the expression of XIAP and emodin downregulated XIAP expression in pancreatic cancer on gene and protein levels. XIAP level decreased more significantly in pancreatic cancer cells in the combination group. XIAP is an important apoptosis-inhibiting protein regulated by NF- $\kappa B$ (28). Studies have found that XIAP mRNA expression level in pancreatic cancer was 2.1 times that of the control in the patient (29). So the mRNA level of XIAP was detected in the tumor tissue in our study. Our results demonstrated XIAP was highly expressed in pancreatic cancer tissue. 
The growth of the tumor can be suppressed by inhibiting XIAP expression in cancer tissue (30). Zou et al demonstrated that the enterocyte apoptosis pathway mediated by surface receptor was blocked through the activation of NF- $\kappa \mathrm{B}$, which can upregulate the expression of XIAP which caused the exhausting of polyamine (28). The inhibition of $N F-\kappa B$ expression not only reduces XIAP expression but also suppresses the proliferation of cancer cells (30). In our study, we observed that emodin alone can inhibit the expression of XIAP and NF- $\kappa \mathrm{B}$ in pancreatic cancer in vitro and in vivo, and the combination of emodin and that gemcitabine was more effective. Our results demonstrated that emodin enhanced antitumor effect of gemcitabine on pancreatic cancer partly by inhibiting the expression of XIAP, which was mediated by inhibiting the expression of NF- $\mathrm{NB}$.

In conclusion, our experiments demonstrated that emodin enhanced antitumor effect of gemcitabine on pancreatic cancer cells by the induction of cell apoptosis in vitro and in vivo. The possible mechanism is that emodin inhibits the upregulation of $\mathrm{NF}-\kappa \mathrm{B}$ induced by gemcitabine and thus inhibits the expression of XIAP in pancreatic cancer cells. XIAP and NF- $\kappa \mathrm{B}$ could become new targets for treatment of pancreatic cancer.

\section{Acknowledgements}

We are grateful for funding support from: Natural Science Foundation of Zhejiang Province, China (Grant No. Y2080708) and the Administration of Traditional Chinese Medicine of Zhengjing Province, China (Grant No. 2011ZZ010). We thank the entire staff of the Animal Experimental Center in Wenzhou Medical College and of scientific research platform of the Second Affiliated Hospital of Wenzhou Medical College for helpful assistance.

\section{References}

1. Jemal A, Siegel R, Xu J and Ward E: Cancer statistics, 2010. CA Cancer J Clin 60: 277-300, 2010.

2. Hidalgo M: Pancreatic cancer. N Engl J Med 362: 1605-1617, 2010.

3. Jemal A, Siegel R, Ward E, Hao Y, Xu J and Thun MJ: Cancer statistics, 2009. CA Cancer J Clin 59: 225-249, 2009.

4. Reni M, Cordio S, Milandri C, et al: Gemcitabine versus cisplatin, epirubicin, fluorouracil, and gemcitabine in advanced pancreatic cancer: a randomised controlled multicentre phase III trial Lancet Oncol 6: 369-376, 2005.

5. Wang XY, Li HJ, Wen H, Yan D and Peng SY: Influence of the adjuvant therapy on the survival of patients with stage II pancreatic carcinoma. Front Med China 4: 430-435, 2010.

6. Kunnumakkara AB, Guha S, Krishnan S, Diagaradjane P, Gelovani J and Aggarwal BB: Curcumin potentiates antitumor activity of gemcitabine in an orthotopic model of pancreatic cancer through suppression of proliferation, angiogenesis, and inhibition of nuclear factor-kappaB-regulated gene products. Cancer Res 67: 3853-3861, 2007.

7. Banerjee S, Kaseb AO, Wang Z, et al: Antitumor activity of gemcitabine and oxaliplatin is augmented by thymoquinone in pancreatic cancer. Cancer Res 69: 5575-5583, 2009.

8. Basu S, Ghosh A and Hazra B: Evaluation of the antibacterial activity of Ventilago madraspatana Gaertn., Rubia cordifolia Linn. and Lantana camara Linn.: isolation of emodin and physcion as active antibacterial agents. Phytother Res 19: 888-894, 2005.

9. Li HL, Chen HL, Li H, et al: Regulatory effects of emodin on NF-kappaB activation and inflammatory cytokine expression in RAW 264.7 macrophages. Int J Mol Med 16: 41-47, 2005.
10. Lin SZ, Chen KJ, Tong HF, Jing H, Li H and Zheng SS: Emodin attenuates acute rejection of liver allografts by inhibiting hepatocellular apoptosis and modulating the Th1/Th2 balance in rats. Clin Exp Pharmacol Physiol 37: 790-794, 2010.

11. Ko JC, Su YJ, Lin ST, Jhan JY, Ciou SC, Cheng CM and Lin YW: Suppression of ERCC1 and Rad51 expression through ERK1/2 inactivation is essential in emodin-mediated cytotoxicity in human non-small cell lung cancer cells. Biochem Pharmacol 79: 655-664, 2010.

12. Li J, Liu P, Mao H, Wanga A and Zhang X: Emodin sensitizes paclitaxel-resistant human ovarian cancer cells to paclitaxelinduced apoptosis in vitro. Oncol Rep 21: 1605-1610, 2009.

13. Wang CG, Yang JQ, Liu BZ, et al: Anti-tumor activity of emodin against human chronic myelocytic leukemia K562 cell lines in vitro and in vivo. Eur J Pharmacol 627: 33-41, 2010.

14. Chen H, Wei W, Guo Y, et al: Enhanced effect of gemcitabine by emodin against pancreatic cancer in vivo via cytochrome C-regulated apoptosis. Oncol Rep 25: 1253-1261, 2011.

15. Huang Q, Shen HM, Shui G, Wenk MR and Ong CN: Emodin inhibits tumor cell adhesion through disruption of the membrane lipid Raft-associated integrin signaling pathway. Cancer Res 66: 5807-5815, 2006.

16. Muto A, Hori M, Sasaki Y, et al: Emodin has a cytotoxic activity against human multiple myeloma as a Janus-activated kinase 2 inhibitor. Mol Cancer Ther 6: 987-994, 2007.

17. Mitsiades N, Mitsiades CS, Poulaki V, et al: Biologic sequelae of nuclear factor-kappaB blockade in multiple myeloma: therapeutic applications. Blood 99: 4079-4086, 2002.

18. Lee KB, Kim KR, Huh TL and Lee YM: Proton induces apoptosis of hypoxic tumor cells by the p53-dependent and p38/JNK MAPK signaling pathways. Int J Oncol 33: 1247-1256, 2008.

19. Pratesi G, Petrangolini G, Tortoreto M, et al: Therapeutic synergism of gemcitabine and CpG-oligodeoxynucleotides in an orthotopic human pancreatic carcinoma xenograft. Cancer Res 65: 6388-6393, 2005.

20. Cha TL, Qiu L, Chen CT, Wen Y and Hung MC: Emodin downregulates androgen receptor and inhibits prostate cancer cell growth. Cancer Res 65: 2287-2295, 2005.

21. Tang ZY, Wu YL, Gao SL and Shen HW: Effects of the proteasome inhibitor bortezomib on gene expression profiles of pancreatic cancer cells. J Surg Res 145: 111-123, 2008.

22. Zhao C, Chen Z, Ye X, et al: Imaging a pancreatic carcinoma xenograft model with 11C-acetate: a comparison study with 18F-FDG. Nucl Med Commun 30: 971-977, 2009.

23. Niu J, Li Z, Peng B and Chiao PJ: Identification of an autoregulatory feedback pathway involving interleukin-lalpha in induction of constitutive NF-kappaB activation in pancreatic cancer cells. J Biol Chem 279: 16452-16462, 2004.

24. Wang W, Abbruzzese JL, Evans DB, Larry L, Cleary KR and Chiao PJ: The nuclear factor-kappa B RelA transcription factor is constitutively activated in human pancreatic adenocarcinoma cells. Clin Cancer Res 5: 119-127, 1999.

25. Vucic D and Fairbrother WJ: The inhibitor of apoptosis proteins as therapeutic targets in cancer. Clin Cancer Res 13: 5995-6000, 2007.

26. Liu A, Chen $\mathrm{H}$, Tong $\mathrm{H}$, et al: Emodin potentiates the antitumor effects of gemcitabine in pancreatic cancer cells via inhibition of nuclear factor-kappaB. Mol Med Rep 4: 221-227, 2011.

27. Guo Q, Chen Y, Zhang B, Kang M, Xie Q and Wu Y: Potentiation of the effect of gemcitabine by emodin in pancreatic cancer is associated with survivin inhibition. Biochem Pharmacol 77: 1674-1683, 2009.

28. Zou T, Rao JN, Guo X, et al: NF-kappaB-mediated IAP expression induces resistance of intestinal epithelial cells to apoptosis after polyamine depletion. Am J Physiol Cell Physiol 286: C1009-C1018, 2004.

29. Shrikhande SV, Kleeff J, Kayed H, et al: Silencing of X-linked inhibitor of apoptosis (XIAP) decreases gemcitabine resistance of pancreatic cancer cells. Anticancer Res 26: 3265-3273, 2006.

30. Deveraux QL, Roy N, Stennicke HR, et al: IAPs block apoptotic events induced by caspase- 8 and cytochrome $\mathrm{c}$ by direct inhibition of distinct caspases. EMBO J 17: 2215-2223, 1998. 